\title{
Treatment outcomes of systemic chemotherapy for peritoneal carcinomatosis arising from gastric cancer with no measurable disease: retrospective analysis from a single center
}

\author{
Sook Hee Hong • Yu Ri Shin · Sang Young Roh • \\ Eun Kyoung Jeon • Kyo Yung Song • Cho Hyun Park • \\ Hae Myung Jeon • Young Seon Hong
}

Received: 11 April 2012/ Accepted: 7 July 2012/Published online: 17 August 2012

(c) The International Gastric Cancer Association and The Japanese Gastric Cancer Association 2012

\begin{abstract}
Background Few studies of systemic chemotherapy have focused on gastric cancer with peritoneal carcinomatosis (PC) without measurable lesions. In the present study, we characterized the outcomes of systemic chemotherapy and prognostic factors for gastric cancer with PC, particularly in patients without measurable disease.

Methods Clinical data from 211 gastric cancer patients with PC (137 without and 74 with measurable disease) who had received systemic chemotherapy between January 2003 and December 2010 at a single center were reviewed. Results The median overall survival (OS) rate of gastric cancer patients with PC with no measurable disease was significantly longer than that of patients with measurable disease (18.0 vs. 11.6 months, $p=0.010)$. On multivariate analysis, poor performance status [hazard ratio $(\mathrm{HR})=$ $2.15, p<0.001]$, the presence of metastatic lymphadenopathy $(\mathrm{HR}=2.17, p<0.001)$, and high-grade $\mathrm{PC}$
\end{abstract}

S. H. Hong · S. Y. Roh · E. K. Jeon · Y. S. Hong $(\bowtie)$

Division of Medical Oncology, Department of Internal

Medicine, Seoul St. Mary's Hospital, The Catholic University of

Korea, 505 Banpo-Dong, Seocho-Gu, Seoul 137-701,

South Korea

e-mail: ssuki76@catholic.ac.kr

S. H. Hong · Y. R. Shin · S. Y. Roh · E. K. Jeon ·

K. Y. Song · C. H. Park · H. M. Jeon · Y. S. Hong

Gastric Cancer Multidisciplinary team, Seoul St. Mary's

Hospital, The Catholic University of Korea, Seoul, South Korea

\section{Y. R. Shin}

Department of Radiology, Incheon St Mary's Hospital,

The Catholic University of Korea, Seoul, South Korea

K. Y. Song · C. H. Park · H. M. Jeon

Department of Surgery, Seoul St. Mary's Hospital,

The Catholic University of Korea, Seoul, South Korea
(HR $=1.83, p=0.001)$ were associated with significantly decreased OS. When patients with low-grade PC were stratified by clinical PC grade, the median OS of those without measurable disease was 19.6 months. The median OS of patients with low-grade PC with no measurable disease was longer than those of patients with high-grade PC without measurable disease, patients with low-grade PC with measurable disease, and patients with high-grade PC with measurable disease $(p=0.001, p=0.029$, and $p<0.001$, respectively). Among the patients with lowgrade PC, patients who received a gastrectomy had longer survival than patients who did not receive a gastrectomy $(p<0.001)$.

Conclusions In our study, clinically low-grade PC without measurable disease was associated with better outcomes of systemic chemotherapy than the outcomes in the other groups examined. Clinical trials in patients with gastric cancer with PC should be stratified according to PC grade.

Keywords Gastric cancer - Peritoneal carcinomatosis Measurable disease $\cdot$ Systemic chemotherapy

\section{Introduction}

About $10-20 \%$ of patients with gastric cancer develop peritoneal dissemination. Furthermore, $60 \%$ of patients with T3 or T4 tumor develop peritoneal recurrence after curative resection [1]. Peritoneal dissemination is considered an independent factor for poor prognosis [2]. While many treatment strategies have been developed to manage peritoneal carcinomatosis (PC) derived from gastric cancer, the outcomes remain poor. A recent large-scale study of PC managed with cytoreductive surgery and perioperative intraperitoneal chemotherapy showed that the median 
overall survival (OS) of patients with PC derived from gastric cancer was only 7-15 months [3, 4]. Even with complete cytoreductive surgery, the 2-year survival rate of patients with, P3 grade PC derived from gastric cancer, classified by Japanese classification of gastric carcinoma, was only $2 \%$ [5].

Several clinical trials of newly developed chemotherapeutic drugs have been conducted. However, few studies have been performed in patients with gastric cancer with PC. Furthermore, it is difficult to include patients with no measurable disease in such clinical trials. In some phase 3 clinical trials, these patients comprised only $5-20 \%$ of the overall patient population [6,7]. Subgroup analysis suggested that patients without measurable disease had somewhat better survival than those with measurable disease, although the presence of PC was a poor prognostic factor [7]. Recently, the effect of systemic chemotherapy on colorectal cancer with PC was retrospectively analyzed. However, to our knowledge, no information on the effect of systemic chemotherapy on gastric cancer with $\mathrm{PC}$ is available at present [8]. In the present study, we characterized the outcomes of systemic chemotherapy and prognostic factors for patients with PC from gastric cancer, with a particular focus on patients without measurable disease.

\section{Patients, materials, and methods}

Patients and methods

After approval was obtained from the Institutional Review Board, patients diagnosed with recurrent or metastatic gastric cancer and treated at Seoul St. Mary's hospital between January 2003 and December 2010 were identified through the cancer registry. Among the patients with recurrent and metastatic gastric cancer, those who met the following criteria were included: (1) pathologically confirmed gastric adenocarcinoma; (2) PC diagnosed with abdominopelvic computed tomodensitometry (CT) scan or surgery; (3) no other distant metastasis, such as liver, lung, or bone; and no extraabdominal metastatic lymphadenopathy (LAP), such as supraclavicular LAP, diagnosed with abdominopelvic CT scan, whole-body bone scan, and/or positron emission tomography CT scan. (4) All patients had normal bone marrow, hepatic, and renal functions. (5) All patients received systemic chemotherapy. (6) Response assessment was performed every 2-3 cycles, based on the Response Evaluation Criteria in Solid Tumor (RECIST) version 1.0 criteria, depending on the chemotherapeutic regimen. (7) Disease progression of evaluable disease (no measurable lesion) was assessed using RECIST criteria [9].

The TNM classification of the American Joint Committee on Cancer staging version 6.0 was used. Clinical staging was applied in cases where pathologic staging was unavailable. A gastrointestinal radiologist (Y. R. Shin) retrospectively reviewed each initial $\mathrm{CT}$ scan for the presence of measurable disease, using RECIST version 1.0 criteria, and if the primary lesion included a measurable lesion, determined whether it satisfied the RECIST version 1.0 criteria [9]. Clinical characteristics of $\mathrm{PC}$ at the initial abdominopelvic CT scan, such as peritoneal thickening or enhancement, either smooth or nodular; small and large bowel involvement with wall thickening and bowel distension; involvement of the omentum, such as soft-tissue permeation of fat, enhancing nodules, or omental cake; and the presence of ascites were retrospectively reviewed and graded. We selected overall survival (OS) and progressionfree survival (PFS) as the main parameters of clinical outcome, because reports of the response rate (RR) were limited, owing to the difficulty of evaluating the response and the difficulty of assessing the RR. After the chemotherapy was completed, disease status was evaluated with abdominopelvic CT, every 3 months if patients were asymptomatic, or when they developed symptoms.

\section{Statistical methods}

The frequencies and descriptive statistics of demographic and clinical variables were determined. The $\chi^{2}$ test and Fisher's exact test were used for categorical variables and Student's $t$-test was used for continuous and ordinal variables, as appropriate. The OS time was calculated from the date of diagnosis to date of death or last known follow up. The PFS time was calculated from the date of diagnosis to the first documentation of disease recurrence or death or known follow up. The survival times were estimated using the Kaplan-Meier method, and the log-rank test was employed to compare survival curves. Univariate and multivariate predictors of OS and PFS were determined using the Cox proportional hazards model. Rate ratios and their $95 \%$ confidence intervals (CIs) were calculated. The SPSS program for Windows (ver. 15.0; SPSS, Chicago, IL, USA) was used for analysis.

\section{Results}

Patient characteristics

Overall, 211 gastric adenocarcinoma patients with PC were identified from the cancer registry. Median follow-up duration was 15.0 months (range 1.5-75.3 months), and 24 $(11.3 \%)$ patients were lost to follow up. Of the 211 patients, 137 had no measurable disease and 74 had one or more measurable lesions. The disease status was classified into three groups: recurrent disease, PC with gastrectomy, 
and PC without gastrectomy. Palliative gastrectomy alone or D1/D2 lymph node (LN) dissection was performed for patients in the PC with gastrectomy group. The patients' baseline characteristics are summarized in Table 1. Gastrectomy alone or D1/D2 LN dissection was more commonly performed in patients without measurable disease (59.0 vs. $32.5 \%, p<0.001)$, because $31.7 \%$ of the patients without measurable disease were diagnosed with $\mathrm{PC}$ during the operation.

Classification of peritoneal carcinomatosis based on clinical characteristics

Among the 137 patients with no measurable disease, 58 $(42.3 \%)$ displayed only peritoneal seeding, nodules, or infiltrations, without ascites or bowel wall involvement. Among the 74 patients with ascites, 16 patients had symptomatic ascites (e.g., abdominal distension, bloating sensation) and required paracentesis $(>500 \mathrm{ml})$. Seventyfour patients underwent the management of gastric outlet obstruction during overall follow-up period. Among them, 8 patients had placement of a gastric stent and 11 patients had a palliative gastrojejunostomy; these 19 patients were classified as having bowel wall involvement. Sixteen $(7.6 \%)$ of the overall 211 patients had a Krukenberg tumor at the time of diagnosis (Table 2). At the time of diagnosis, 2 patients had a total hysterectomy with bilateral salpingooophorectomy and 1 patient had an oophorectomy only.

In terms of clinical characteristics, PC was classified into three grades. Grade 0 was defined as peritoneal seeding detected during operation with no evidence of $\mathrm{PC}$ in the preoperative evaluation, and grade 1 was defined as peritoneal seeding or ascites detected from an abdominopelvic CT scan; however, no bowel involvement or need for paracentesis was recorded. Grade 2 was defined as bowel wall involvement (gastric, small bowel, and large bowel wall) or a large amount of ascites requiring paracentesis. According to this carcinomatosis grading system, 39 (28.5\%), 48 (35.0\%), and $50(36.5 \%)$ of the 137 patients without measurable disease and $9(12.2 \%), 40(54.1 \%)$, and $25(33.8 \%)$ of the 74 patients with measurable disease were classified as grades 0,1 , and 2, respectively (Table 2).

Based on the clinical characteristics and the lack of difference in survival rates, grades 0 and 1 were summed as low-grade and grade 2 was defined as high-grade (Table 2). Those with low-grade PC showed better performance status (PS) than those with high-grade disease, and larger numbers of patients received gastrectomy and second-line chemotherapy compared with those with high-grade disease ( $p<0.001, p<0.001$, and $p=0.027$, respectively). In terms of metastatic LAP, $56.6 \%$ of the patients with low-grade PC and $42.7 \%$ of the patients with high-grade PC had metastatic LAP. This finding may have been related to the more frequent surgical staging and diagnosis of the patients with low-grade disease during surgery. This finding also reflects the discrepancy between surgical staging and preoperative staging in the detection of metastatic LAP. The mean dose of chemotherapy for low-grade PC was higher than that for high-grade PC $(p=0.009)$ and more patients with low-grade PC than patients with highgrade disease received second line-chemotherapy (80.9 vs. $64.8 \%, p=0.027$ ) (Table 3).

Survival of patients with no measurable disease, according to clinical characteristics

The median OS and PFS of patients without measurable disease were 18.6 months (95\% CI 15.9-20.5 months) and 8.27 months (95\% CI 7.3-9.2 months), respectively. On univariate analysis, PS, presence of metastatic LAP, disease status, and carcinomatosis grade had significant effects on PFS and OS. On multivariate analysis, poor PS $(\mathrm{HR}=2.15,95 \%$ CI $1.36-3.41, p<0.001)$, metastatic LAP $(\mathrm{HR}=2.17,95 \%$ CI 1.43-3.30, $p<0.001)$, and high-grade $\mathrm{PC}(\mathrm{HR}=1.83,95 \%$ CI $1.43-3.30, p=0.01)$ were associated with significantly decreased OS rates. Additionally, PFS rates were significantly decreased in patients with poor PS $(\mathrm{HR}=1.63,95 \%$ CI $1.43-3.30$, $p=0.023)$, metastatic LAP $(\mathrm{HR}=2.09,95 \%$ CI $1.42-3.07, p<0.001)$, and high-grade $\mathrm{PC}(\mathrm{HR}=1.53$, $95 \%$ CI $1.00-2.32, p=0.047$ ) (Table 4 ).

The clinical characteristics of PC at the time of progression after first-line chemotherapy

Among the 137 PC patients without measurable disease, the disease status of 131 could be evaluated at the time of progressive disease after first-line chemotherapy. After progression, $92(67.2 \%)$ patients still had no measurable disease. The most common cause of progressive disease was increased or newly developed ascites $(n=25$, $18.2 \%$ ) (Table 5). Of the entire cohort of 211 patients, 149 patients received second-line chemotherapy, 51 patients did not receive second-line chemotherapy, and 11 patients did not progress on first-line chemotherapy. The median OS of the patients who received the second-line chemotherapy was 17.3 months (95\% CI $14.3-20.4$ months) and that of the patients who did not receive the second-line chemotherapy was 7.3 months $(95 \% \mathrm{CI}$ 5.6-9.1 months, $p<0.001$ ). Among the overall 51 patients who did not receive second line chemotheraphy common reasons for patients not receiving second-line chemotherapy were bowel obstruction $22(43.1 \%)$, patient's refusal 8 $(15.7 \%)$, decreased PS 7 (13.7 \%), and an infectious condition $6(11.7 \%)$. 
Table 1 Clinical characteristics of patients with gastric cancer peritoneal carcinomatosis (PC)

\begin{tabular}{|c|c|c|c|c|}
\hline & $\begin{array}{l}\text { All patients } \\
(n=211) \\
\text { No. }(\%)\end{array}$ & $\begin{array}{l}\text { Measurable disease } \\
(n=74) \\
\text { No. }(\%)\end{array}$ & $\begin{array}{l}\text { No measurable disease } \\
(n=74) \\
\text { No. }(\%)\end{array}$ & $\begin{array}{l}\text { Measurable versus } \\
\text { no measurable disease } \\
P\end{array}$ \\
\hline Age, years mean (range) & $54.5(22-81)$ & $55.2(33-78)$ & $54.1(22-81)$ & \\
\hline \multicolumn{5}{|l|}{ Sex } \\
\hline Female & $120(56.9 \%)$ & $56(75.7 \%)$ & $64(46.7 \%)$ & \multirow[t]{2}{*}{0.176} \\
\hline Male & $91(43.1 \%)$ & $18(24.3 \%)$ & $73(53.3 \%)$ & \\
\hline \multicolumn{5}{|l|}{ Performance status } \\
\hline $0-1$ & $128(60.6 \%)$ & $46(62.2 \%)$ & $82(59.9 \%)$ & \multirow[t]{2}{*}{0.855} \\
\hline$\geq 2$ & $79(37.4 \%)$ & $27(36.5 \%)$ & $52(37.9 \%)$ & \\
\hline \multicolumn{5}{|l|}{ Histologic subtype } \\
\hline WD and MD & $40(19.0 \%)$ & $18(24.4 \%)$ & $22(15.8 \%)$ & \multirow[t]{5}{*}{0.082} \\
\hline PD & $127(60.2 \%)$ & $62(83.8 \%)$ & $65(61.3 \%)$ & \\
\hline Mucinous & $4(1.9 \%)$ & $1(1.4 \%)$ & $3(20.4 \%)$ & \\
\hline Signet ring cell & $38(18 \%)$ & $10(13.5 \%)$ & $28(2.2 \%)$ & \\
\hline Unclassifiable & $2(0.9 \%)$ & $2(2.7 \%)$ & $0(\%)$ & \\
\hline \multicolumn{5}{|l|}{ Borrmann type } \\
\hline I and II & $15(7.1 \%)$ & $4(5.4 \%)$ & $11(8.0 \%)$ & \multirow[t]{4}{*}{0.098} \\
\hline III & $105(49.8 \%)$ & $45(60.8 \%)$ & $60(43.8 \%)$ & \\
\hline IV & $78(37 \%)$ & $19(25.7 \%)$ & $59(43.1 \%)$ & \\
\hline Others & $13(6.1 \%)$ & $6(8.1 \%)$ & $7(5.1 \%)$ & \\
\hline \multicolumn{5}{|l|}{ Metastatic LAP } \\
\hline Yes & $110(52.1 \%)$ & $56(75.7 \%)$ & $54(39.4 \%)$ & \multirow[t]{2}{*}{$<0.001$} \\
\hline No & $101(47.9 \%)$ & $18(24.3 \%)$ & $83(60.6 \%)$ & \\
\hline \multicolumn{5}{|l|}{ Disease status } \\
\hline Recurrent & $60(28.4 \%)$ & $19(25.7 \%)$ & $41(29.9 \%)$ & \multirow[t]{3}{*}{$<0.001$} \\
\hline PC with gastrectomy & $45(21.3 \%)$ & $5(6.8 \%)$ & $40(29.2 \%)$ & \\
\hline PC without gastrectomy & $106(50.3 \%)$ & $50(67.6 \%)$ & $56(40.9 \%)$ & \\
\hline \multicolumn{5}{|l|}{ First-line chemotherapy } \\
\hline 5-FU/platinum & $155(73.5 \%)$ & $57(77.0 \%)$ & $98(71.5 \%)$ & \multirow[t]{5}{*}{0.36} \\
\hline Taxane/CDDP & $25(11.8 \%)$ & $10(13.5 \%)$ & $15(10.9 \%)$ & \\
\hline FOLFIRI & $12(5.7 \%)$ & $3(4.1 \%)$ & $9(6.6 \%)$ & \\
\hline Oral 5-FU & $12(5.7 \%)$ & $2(2.7 \%)$ & $10(7.3 \%)$ & \\
\hline Others & $7(3.3 \%)$ & $2(2.7 \%)$ & $5(3.6 \%)$ & \\
\hline \multicolumn{5}{|c|}{ Number of chemotherapy regimens } \\
\hline 1 & $77(36.3 \%)$ & $24(32.4 \%)$ & $53(38.7 \%)$ & \multirow[t]{3}{*}{0.76} \\
\hline 2 & $63(29.7 \%)$ & $24(32.4 \%)$ & $39(28.5 \%)$ & \\
\hline$\geq 3$ & $71(34.0 \%)$ & $26(35.2 \%)$ & $45(32.8 \%)$ & \\
\hline
\end{tabular}

$W D$ well differentiated, $M D$ moderately differentiated, $P D$ poorly differentiated, $L A P$ lymphadenopathy, $C D D P$ cisplatin, $F U$ fluorouracil, FOLFIRI fluorouracil, leucovorin, irinotecan

Survival of patients with PC stratified according to carcinomatosis grade

The median OS of patients without measurable disease was significantly longer than that of patients with measurable disease (18.0 vs. 11.6 months, $p=0.010$ ) (Fig 1). However, the median OS values in female patients, patients with poor PS, those with Borrmann type IV, and those with recurrent gastric cancer as disease status showed no significant differences between patients with and without measurable disease (Fig. 1; Table 6).

The overall median OS and PFS times of patients with grade $0 \mathrm{PC}$ were significantly longer than those of patients with grade 2 PC $(p<0.001$ and $p<0.001$, respectively). However, no differences in PFS or OS were observed between grade 0 and grade 1 PC (Fig. 2a). Among the patients without measurable disease, the median OS and PFS times of patients with grade 0 PC were significantly 
Table 2 Findings and grading of gastric cancer peritoneal carcinomatosis

\begin{tabular}{|c|c|c|}
\hline $\begin{array}{l}\text { Findings of the peritoneal } \\
\text { carcinomatosis }\end{array}$ & $\begin{array}{l}\text { Measurable } \\
\text { disease } \\
(n=74) \\
\text { No. }(\%)\end{array}$ & $\begin{array}{l}\text { No measurable } \\
\text { disease } \\
(n=137) \\
\text { No. }(\%)\end{array}$ \\
\hline Peritoneal seeding & $36(48.6 \%)$ & $58(42.3 \%)$ \\
\hline No symptomatic ascites & $32(4.5 \%)$ & $58(42.3 \%)$ \\
\hline $\begin{array}{l}\text { Gastric outlet obstruction } \\
\text { (no gastrectomy) }\end{array}$ & $9(12.2 \%)$ & $9(6.5 \%)$ \\
\hline Small bowel involvement & $6(8.1 \%)$ & $15(10.9 \%)$ \\
\hline Large bowel involvement & $10(11.9 \%)$ & $37(27.0 \%)$ \\
\hline $\begin{array}{l}\text { Large amount ascites needed } \\
\text { paracentesis }\end{array}$ & $7(9.5 \%)$ & $16(11.7 \%)$ \\
\hline Krukenberg tumor & $11(14.9 \%)$ & $5(3.6 \%)$ \\
\hline \multicolumn{3}{|l|}{ Peritoneal carcinomatosis grading } \\
\hline \multicolumn{3}{|l|}{ Low } \\
\hline $\begin{array}{l}0 \text { Peritoneal seeding detected } \\
\text { during surgery }\end{array}$ & $9(12.2 \%)$ & $39(28.5 \%)$ \\
\hline $\begin{array}{l}1 \text { Ascites or peritoneal } \\
\text { seeding without bowel } \\
\text { involvement }\end{array}$ & $40(54.1 \%)$ & $48(35.0 \%)$ \\
\hline \multicolumn{3}{|l|}{ High } \\
\hline $\begin{array}{l}2 \text { Large amount ascites } \\
\text { or bowel involvement }\end{array}$ & $25(33.8 \%)$ & $50(36.5 \%)$ \\
\hline
\end{tabular}

longer than the median OS and PFS times of patients with grade 2 PC ( $p=0.001$ and $p=0.003$, respectively). No differences in PFS or OS were observed between grade 0 and grade 1 PC (Fig. 2b).

We summed grades 0 and 1 as the low-grade PC group, owing to the lack of difference in their survival rates, and we classified grade 2 as high-grade PC. All patients were then divided into 4 groups: low-grade PC without measurable disease, high-grade PC without measurable disease, lowgrade $\mathrm{PC}$ with measurable disease, and high-grade $\mathrm{PC}$ with measurable disease. The median OS time of patients with lowgrade $\mathrm{PC}$ without measurable disease was significantly longer than those of the patients in the three other groups ( $p=0.001$, $p=0.029$, and $p<0.001$, respectively) (Fig. 3a). The median PFS of the low-grade PC group without measurable disease was also significantly longer than those of the patients from the three other groups $(p=0.010, p<0.0 .001$, and $p<0.001$, respectively) (Fig. 3b). We observed no differences in median OS and PFS between high-grade PC with measurable disease and low-grade PC without measurable disease ( $p=0.281$ and 0.592 , respectively).

Survival of patients with low-grade PC stratified according to gastrectomy status

Among the 127 patients with low-grade $\mathrm{PC}$ at the time of diagnosis (disease not recurrent), the median OS of patients
Table 3 Clinical characteristics of peritoneal carcinomatosis according to the carcinomatosis grade

\begin{tabular}{|c|c|c|c|}
\hline & $\begin{array}{l}\text { Low-grade } \\
\text { carcinomatosis } \\
(n=136) \\
\text { No. }(\%)\end{array}$ & $\begin{array}{l}\text { High-grade } \\
\text { carcinomatosis } \\
(n=75) \\
\text { No. }(\%)\end{array}$ & $P$ \\
\hline \multicolumn{4}{|l|}{ Sex } \\
\hline Male/female & $\begin{array}{l}72(52.9 \%) / \\
65(47.1 \%)\end{array}$ & $\begin{array}{l}48(64.0 \%) / \\
26(36.0 \%)\end{array}$ & 0.109 \\
\hline PS & & & $<0.001$ \\
\hline $0-1 / \geq 2$ & $\begin{array}{l}99(72.8 \%) / \\
35(25.7 \%)\end{array}$ & $\begin{array}{l}29(38.6 \%) / \\
43(57.3 \%)\end{array}$ & \\
\hline \multicolumn{4}{|l|}{ Metastatic LAP } \\
\hline Yes/no & $77(56.6 \%)$ & $32(42.7 \%)$ & 0.052 \\
\hline \multicolumn{4}{|l|}{ Histologic subtype } \\
\hline WD and MD & $22(16.1 \%)$ & $5(6.7 \%)$ & 0.053 \\
\hline PD and signet ring cell & $110(80.9 \%)$ & $68(90.7 \%)$ & \\
\hline \multicolumn{4}{|l|}{ Borrmann type } \\
\hline I-III/IV & $\begin{array}{l}85(61.2 \%) / \\
54(38.8 \%)\end{array}$ & $\begin{array}{l}34(54.8 \%) / \\
21(45.2 \%)\end{array}$ & 0.535 \\
\hline \multicolumn{4}{|l|}{ Disease status } \\
\hline Recurrent & $20(14.7 \%)$ & $37(49.3 \%)$ & $<0.001$ \\
\hline $\mathrm{PC}$ with gastrectomy & $44(32.3 \%)$ & & \\
\hline $\begin{array}{l}\text { PC without } \\
\text { gastrectomy }\end{array}$ & $72(53.0 \%)$ & $38(50.7 \%)$ & \\
\hline $\begin{array}{l}\text { Mean dose of first-line } \\
\text { chemotherapy }\end{array}$ & $95.8 \%$ & $86.1 \%$ & 0.009 \\
\hline $\begin{array}{l}\text { Delayed chemotherapy } \\
\text { more than } 2 \text { weeks }\end{array}$ & $62(45.3 \%)$ & $19(25.6 \%)$ & 0.782 \\
\hline $\begin{array}{l}\text { Duration of first-line } \\
\text { chemotherapy }\end{array}$ & 160 days & 94 days & 0.057 \\
\hline $\begin{array}{l}\text { No. of first-line } \\
\text { chemotherapy cycles }\end{array}$ & 7.5 & 6.6 & 0.245 \\
\hline $\begin{array}{l}\text { No. of chemotherapy } \\
\text { regimens }\end{array}$ & 2.3 & 2.0 & 0.229 \\
\hline $\begin{array}{l}\text { Second-line } \\
\text { chemotherapy }\end{array}$ & & & 0.027 \\
\hline Yes & $102(80.9 \%)$ & $46(64.8 \%)$ & \\
\hline No & $26(19.1 \%)$ & $25(35.2 \%)$ & \\
\hline
\end{tabular}

$P C$ peritoneal carcinomatosis

with low-grade PC with gastrectomy (29.3 months, $95 \%$ CI 17.9-40.7) was longer than that of the patients without gastrectomy (12.5 months, $95 \%$ CI 9.6-15.4, $p<0.001)$ (Fig. 4). Among the patients with low-grade PC, both grade 0 and grade 1 PC patients with gastrectomy showed longer survival than the patients without gastrectomy ( $p=0.001$ and $p=0.021$, respectively) (Fig. 5). However, for patients with low-grade PC with measurable disease, there was no difference in median OS according to gastrectomy status $(p=0.604)$. 
Table 4 Univariate and multivariate analyses for overall survival (OS) and progressionfree survival (PFS) of patients with gastric cancer peritoneal carcinomatosis without measurable disease
$H R$ hazard ratio, $C I$ confidence interval
OS, months, median $(95 \% \mathrm{CI})$

$P$

PFS, months, median (95\% CI)

Univariate analysis

Age, years

$<55$

$\geq 55$

$18.0(14.2-21.8)$

$18.2(14.9-21.4)$

$19.6(17.1-22.2)$

Female

$15.6(10.4-20.8)$

Male

$22.0(18.4-26.0)$

$<0.001$

$13.4(6.8-20.1)$

$\geq 2$

$22.0(14.5-26.9)$

$16.6(11.7-21.4)$

$17.8(6.7-28.8)$

Signet ring cell

Borrmann type

$\begin{array}{ll}\text { I-III } & 19.5(15.0-24.0)\end{array}$

IV

$16.8(10.9-22.7)$

Metastatic LAP

Yes $\quad 14.0(9.7-18.3)$

No

$19.2(17.6-20.8)$

Gastric outlet obstruction

$\begin{array}{ll}\text { Yes } & 5.3(1.9-8.7) \\ \text { No } & 8.3(7.3-9.3)\end{array}$

Disease status

$\begin{array}{ll}\text { Recurrent } & 16.4(8.5-24.4) \\ \text { PC with gastrectomy } & 22.4(11.4-33.5) \\ \text { PC without gastrectomy } & 11.9(8.4-15.4)\end{array}$

First-line chemotherapy

$\begin{array}{ll}\text { 5-FU/platinum } & 16.6(12.7-20.5) \\ \text { Others } & 19.2(17.4-21.0)\end{array}$

Route of first-line chemotherapy

$$
\text { Oral } 14.6(9.3-19.8)
$$

Intravenous

$18.9(17.2-20.7)$

\begin{tabular}{|c|c|c|c|c|}
\hline Low & $19.6(15.8-23.5)$ & $<0.001$ & $8.9(7.8-10.0)$ & 0.01 \\
\hline High & $12.2(9.2-15.2)$ & & $6.7(5.0-8.5)$ & \\
\hline
\end{tabular}

Carcinomatosis grade

\begin{tabular}{|c|c|c|c|c|}
\hline & HR for OS $(95 \% \mathrm{CI})$ & $P$ & HR for PFS $(95 \% \mathrm{CI})$ & $P$ \\
\hline \multicolumn{5}{|c|}{ Multivariate analysis } \\
\hline \multicolumn{5}{|c|}{ Performance status } \\
\hline $0-1$ & 1 & 0.023 & 1 & $<0.001$ \\
\hline$\geq 2$ & $1.63(1.43-3.30)$ & & $2.15(1.36-3.41)$ & \\
\hline \multicolumn{5}{|c|}{ Metastatic LAP } \\
\hline No & 1 & $<0.001$ & 1 & $<0.001$ \\
\hline Yes & $2.09(1.42-3.07)$ & & $2.17(1.43-3.30)$ & \\
\hline \multicolumn{5}{|c|}{ Carcinomatosis grade } \\
\hline Low & 1 & 0.047 & 1 & 0.01 \\
\hline High & $1.53(1.00-2.32)$ & & $1.83(1.43-3.30)$ & \\
\hline
\end{tabular}

\begin{tabular}{|c|c|c|}
\hline 0.963 & $\begin{array}{l}8.1(6.9-9.4) \\
8.3(6.7-9.9)\end{array}$ & 0.650 \\
\hline 0.101 & $\begin{array}{l}8.6(7.3-9.9) \\
7.7(6.1-9.3)\end{array}$ & 0.242 \\
\hline$<0.001$ & $\begin{array}{l}8.9(6.9-11.0) \\
5.6(2.9-8.3)\end{array}$ & 0.001 \\
\hline 0.485 & $\begin{array}{l}9.8(5.9-13.7) \\
8.1(6.5-9.7) \\
7.5(5.7-9.3)\end{array}$ & 0.267 \\
\hline 0.084 & $\begin{array}{l}8.7(7.8-9.7) \\
6.7(5.6-7.8)\end{array}$ & 0.034 \\
\hline 0.044 & $\begin{array}{l}7.2(5.6-8.3) \\
9.0(7.6-10.4)\end{array}$ & 0.001 \\
\hline 0.495 & $\begin{array}{r}7.2(1.1-14.2) \\
18.0(15.2-20.8)\end{array}$ & 0.152 \\
\hline 0.007 & $\begin{array}{c}7.7(5.6-9.8) \\
12.9(9.5-16.3) \\
6.6(4.5-8.7)\end{array}$ & $<0.001$ \\
\hline 0.057 & $\begin{array}{l}8.3(7.2-9.4) \\
7.4(5.1-9.6)\end{array}$ & 0.568 \\
\hline
\end{tabular}

$0.368 \quad 8.1(6.2-9.9) \quad 0.318$

$8.4(7.0-9.9)$

01 .

.


Table 5 Clinical characteristics of progressive disease after first-line chemotherapy in patients with gastric cancer peritoneal carcinomatosis without measurable disease

\begin{tabular}{|c|c|c|}
\hline & $\begin{array}{l}\text { Measurable } \\
\text { disease No. } \\
(\%)\end{array}$ & $\begin{array}{l}\text { No } \\
\text { measurable } \\
\text { disease No. } \\
(\%)\end{array}$ \\
\hline Ascites & & $25(18.2 \%)$ \\
\hline Bowel involvement & $7(5.1 \%)$ & $22(16.0 \%)$ \\
\hline Peritoneal seeding & & $17(12.4 \%)$ \\
\hline Metastatic LAP & $2(1.5 \%)$ & \\
\hline Ascites + bowel involvement & $1(0.7 \%)$ & $5(3.6 \%)$ \\
\hline Ascites + peritoneal seeding & $1(0.7 \%)$ & $7(5.1 \%)$ \\
\hline Ascites + peritoneal seeding + LAP & $4(2.9 \%)$ & \\
\hline Local progression & & $11(8.0 \%)$ \\
\hline Krukenburg tumor & $8(5.8 \%)$ & \\
\hline $\begin{array}{l}\text { Liver/bone metastasis/leptomeningeal } \\
\text { seeding }\end{array}$ & $2(1.5 \%)$ & $3(2.2 \%)$ \\
\hline Others & $4(2.9 \%)$ & $2(1.5 \%)$ \\
\hline Total & $29(21.2 \%)$ & $92(67.2 \%)$ \\
\hline
\end{tabular}



Fig. 1 Kaplan-Meier survival analysis for overall survival $(O S)$ rates of gastric cancer patients with peritoneal carcinomatosis (PC) with no measurable disease compared with rates in the patients with measurable disease. $C I$ confidence interval

\section{Discussion}

The majority of PC patients without measurable disease are not eligible for clinical trials; consequently, the outcomes of systemic chemotherapy in this group of patients are not well known. Our study showed that after disease progression following first-line chemotherapy, $67.2 \%$ of patients with evaluable disease still had no measurable lesion at the initiation of second-line chemotherapy. Moreover, patients without measurable disease showed better OS than those with measurable disease. This finding may be attributed to
Table 6 Median OS rates of patients with gastric cancer peritoneal carcinomatosis without measurable disease compared with the rates in the patients with measurable disease

\begin{tabular}{|c|c|c|c|}
\hline & $\begin{array}{l}\text { Measurable } \\
\text { disease } \\
(n=74) \\
\text { median } \\
(95 \% \mathrm{CI})\end{array}$ & $\begin{array}{l}\text { No measurable } \\
\text { disease } \\
(n=137) \\
\text { median } \\
(95 \% \mathrm{CI})\end{array}$ & $P$ \\
\hline \multicolumn{4}{|l|}{ Age, years } \\
\hline$<55$ & $14.5(4.5-24.4)$ & $18.0(14.2-21.8)$ & 0.119 \\
\hline$\geq 55$ & $12.0(9.3-14.7)$ & $18.2(14.9-21.4)$ & 0.043 \\
\hline \multicolumn{4}{|l|}{ Sex } \\
\hline Female & $13.7(10.0-17.4)$ & $15.6(10.4-20.8)$ & 0.306 \\
\hline Male & $10.9(6.9-14.9)$ & $19.6(17.1-22.2)$ & 0.023 \\
\hline \multicolumn{4}{|l|}{ Performance status } \\
\hline $0-1$ & $13.9(10.3-17.5)$ & $22.2(18.4-26.0)$ & 0.011 \\
\hline$\geq 2$ & $8.0(3.5-12.5)$ & $13.4(6.8-20.1)$ & 0.688 \\
\hline \multicolumn{4}{|l|}{ Histologic subtype } \\
\hline WD and MD & $11.6(9.3-14.0)$ & $22.2(17.5-26.8)$ & 0.070 \\
\hline PD & $13.7(6.8-20.5)$ & $16.6(11.8-21.4)$ & 0.138 \\
\hline Signet ring cell & $17.8(6.7-28.8)$ & $22.7(0.3-57.1)$ & 0.422 \\
\hline \multicolumn{4}{|l|}{ Borrmann type } \\
\hline I-III & $12.5(8.4-16.6)$ & $19.5(15.1-24.0)$ & 0.017 \\
\hline IV & $10.9(6.4-15.5)$ & $16.8(11.0-22.7)$ & 0.278 \\
\hline \multicolumn{4}{|l|}{ Metastatic LAP } \\
\hline Yes & $10.9(8.9-12.9)$ & $14.0(9.7-18.3)$ & 0.145 \\
\hline No & $25.3(16.6-34.0)$ & $26.5(21.3-31.5)$ & 0.945 \\
\hline \multicolumn{4}{|l|}{ Disease status } \\
\hline Recurrent & $13.9(5.0-22.9)$ & $16.4(8.5-24.4)$ & 0.182 \\
\hline PC with gastrectomy & $25.5(0-64.2)$ & $22.4(11.4-33.5)$ & 0.790 \\
\hline $\begin{array}{l}\text { PC without } \\
\text { gastrectomy }\end{array}$ & $10.9(8.9-12.9)$ & $11.9(8.4-15.4)$ & 0.490 \\
\hline \multicolumn{4}{|l|}{ Carcinomatosis grade } \\
\hline Low & $13.9(11.3-16.5)$ & $19.6(15.8-23.4)$ & 0.007 \\
\hline High & $8.4(5.8-11.2)$ & $12.6(8.9-12.3)$ & 0.124 \\
\hline \multicolumn{4}{|c|}{ First-line chemotherapy regimens } \\
\hline 5-FU/platinum & $11.6(9.1-14.2)$ & $16.6(12.7-20.5)$ & 0.079 \\
\hline Others & $13.9(7.5-20.4)$ & $19.2(17.4-21.0)$ & 0.082 \\
\hline
\end{tabular}

the fact that patients without measurable disease had less LN metastasis and a lower disease burden than those with measurable disease. In terms of tumor burden, $29.2 \%$ of our PC patients without measurable disease received gastrectomy alone or D1/D2 LN dissection. None of these patients had any sign of PC during routine preoperative evaluation. Earlier, Yonemura et al. [5] reported that among patients with PC, those receiving cytoreductive surgery showed better survival. Lee and colleagues [10] reported better survival in their resected metastatic and recurrent disease groups than in the initially metastatic group, and suggested that disease status related to tumor burden and not gastrectomy was an important prognostic 

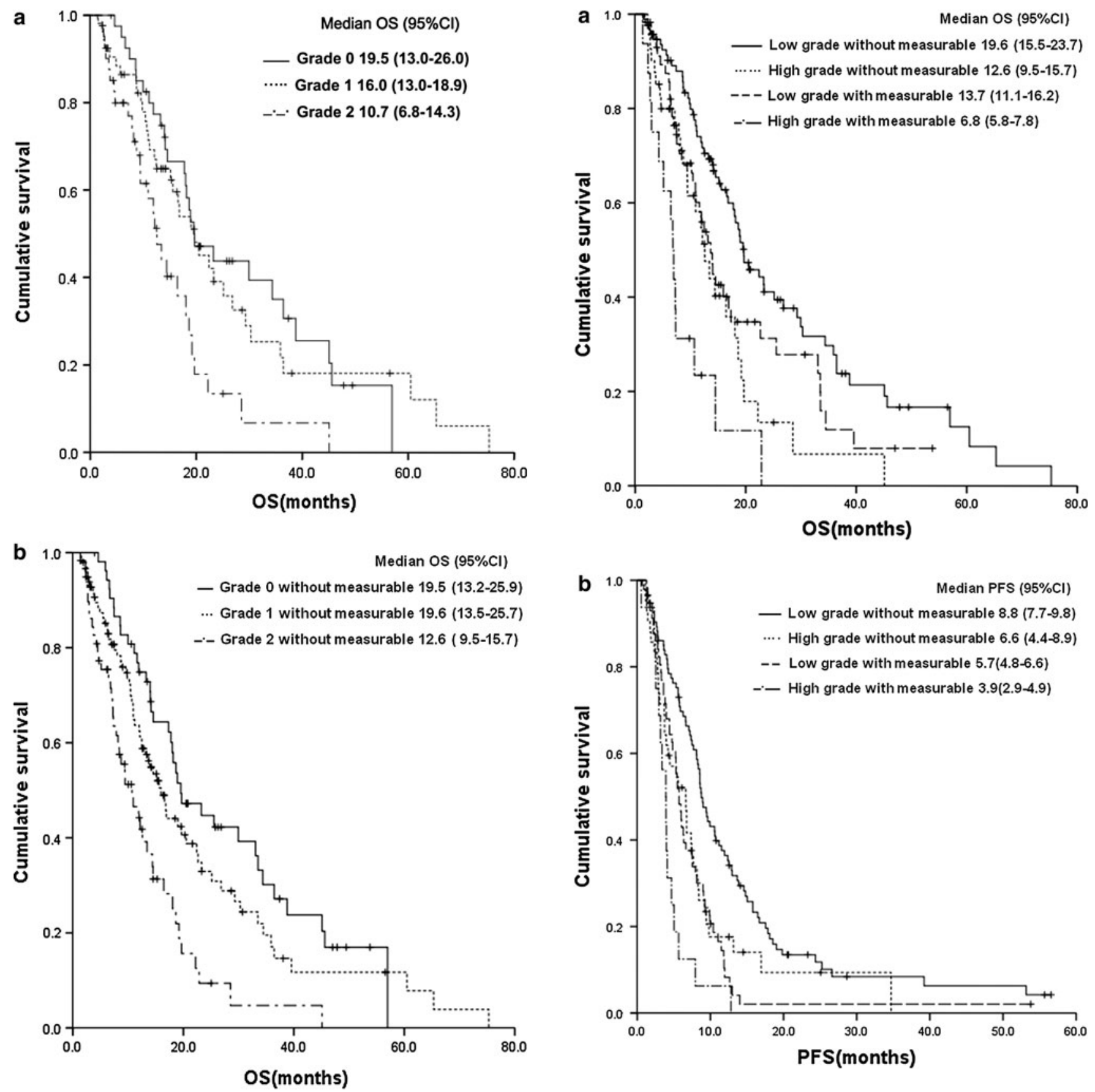

Fig. 2 Kaplan-Meier survival analysis for OS rates of the entire cohort of patients with gastric cancer PC (a) and OS rates in the patients without measurable disease (b) according to the clinical PC grade

factor for gastric cancer. In the present study, $32.3 \%$ of patients with low-grade PC received a gastrectomy, compared with $0 \%$ of those with high-grade PC. Among the patients with low-grade PC, the patients who received a gastrectomy showed better survival than the patients who did not receive a gastrectomy. We thought that some clinical selection biases for gastrectomy could have been

Fig. 3 Kaplan-Meier survival analysis for OS (a) and progressionfree survival $(P F S)(\mathbf{b})$ rates of the patients with gastric cancer PC stratified according to four carcinomatosis grades

present, such as good PS, less likelihood of metastatic LAP, and fewer peritoneal seeding nodules. Thus, the benefit of palliative gastrectomy should be confirmed by prospective clinical trials. The criteria we used for lowgrade PC were similar to the inclusion criteria for the Reductive Gastrectomy for Advanced Tumor in Two Asian countries (REGATTA) trial-a prospective clinical trial of palliative gastrectomy for gastric cancer with limited PC. To define the role of palliative gastrectomy for patients 


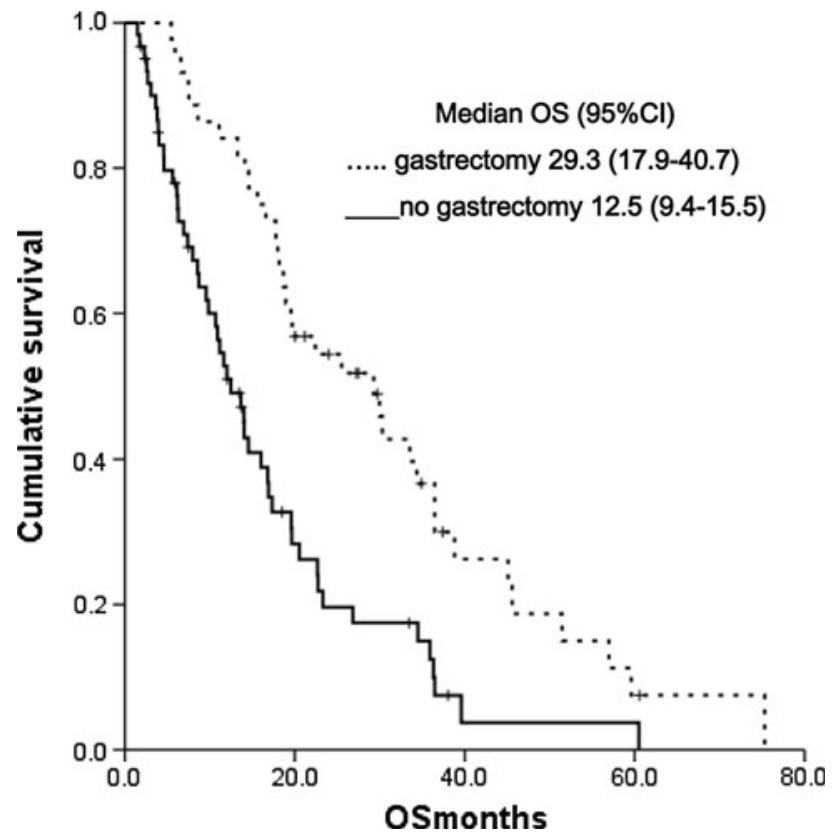

Fig. 4 Kaplan-Meier survival analysis for OS rates of the patients with gastric cancer low-grade PC stratified according to gastrectomy status

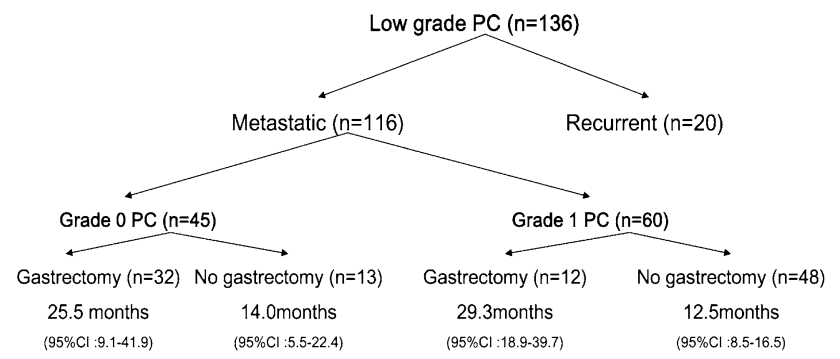

Fig. 5 Differences in OS rates of the patients with low-grade PC according to their gastrectomy status at the time of diagnosis

with gastric cancer with PC, we should wait for the results of this trial.

In terms of tumor burden, several classifications have been applied for PC of gastric cancer. The most commonly used system in Korea and Japan is the Japanese classifcation of gastric carcinoma (stages P1, P2, and P3) [11]. Other systems, such as the PCI (peritoneal cancer index) or Lyon classification, have also been evaluated for gastric cancer PC [3, 10, 12]. These classification stages were determined after surgical exploration. If patients are to receive systemic chemotherapy alone, surgical exploration will promote morbidity and delay systemic chemotherapy. Our simple classification of PC was determined solely on the basis of clinical factors. PC with the presence of ascites requiring paracentesis, or the presence of bowel wall involvement, such as wall thickening, was classified as high-grade. This patient population has already been established as a poor prognosis group [13]. Grade 0, defined as the presence of peritoneal seeding detected during surgery, is another well-known subgroup classified into P1, P2, and P3 stages, based on surgical exploration. Our data further showed that 43.4 and $32.1 \%$ of patients with grade $0 \mathrm{PC}$ were at the $\mathrm{P} 1$ and $\mathrm{P} 2$ stages, respectively. The grade 1 group, defined as those with PC detected with preoperative radiologic imaging without clinically significant ascites and bowel wall involvement, has not been classified according to other grading systems for peritoneal caricnomatosis derived from gastric cancer. This group of patients showed better OS than those classified as grade 2, but no differences in OS compared with grade 0 patients, especially in the patients without measurable disease (19.5 vs. 19.6 months, $p=0.639$ ). In addition, this group of patients (i.e., the grade 1 group) had better outcomes with gastrectomy and systemic chemotherapy than with chemotherapy alone. The classifications and clinical characteristics of PC indicate heterogeneity within gastric cancer PC, and treatment strategies should thus be varied according to the PC grade and molecular characteristics.

The response rates of systemic chemotherapy for gastric cancer with PC with measurable disease were reported to be $14-25 \%$ by a number of phase 2 clinical trials $[14,15]$. The main reason for the poor response rates of systemic chemotherapy in patients with PC was attributed to the existence of the blood peritoneal barrier, which inhibits drug movement [16]. In earlier studies, the efficacy of cytoreductive surgery and intraperitoneal (IP) chemotherapy was evaluated in patients with PC $[3,6]$. The results for gastric cancer were the worst among those for gastrointestinal cancers with PC [3]. In patients with PC who had cytoreductive surgery and IP chemotherapy, Glehen et al. [3] reported that the PCI, completeness of surgery, and LN metastasis were significant prognostic factors for OS. LN metastasis was shown in 117 of their 125 gastric cancer patients with PC [3]. In our present study, $52.1 \%$ of patients had metastatic LAP before systemic chemotherapy was begun. In view of the limitations of the effects of IP chemotherapy for PC with $\mathrm{LN}$ metastasis and the frequent LN metastasis in gastric cancer with PC, other treatment strategies should be employed for this disease [5, 6]. In terms of newer treatment strategies, it has been shown that angiogenesis is important for the development of gastric cancer PC, and the inhibition of angiogenesis is reported to be effective for the management of PC [17-19]. Yagi et al. [20] reported that, in a PC model for gastric cancer, systemic administration of a monoclonal antibody against vascular endothelial growth factor (VEGF) was effective in reducing ascites and inhibiting tumor growth. In addition, systemic administration of the VEGF antibody was more effective than intraperitoneal administration [20]. Subgroup analysis of a phase 3 clinical trial of bevacizumab, a monoclonal antibody against VEGF, revealed a greater 
survival benefit in gastric cancer patients without measurable disease than in those with measurable disease [7]. We hypothesized that a considerable proportion of these patients had PC, and that bevacizumab had led to the normalization of abnormal tumor blood vessels and increased the delivery of systemic chemotherapeutic drugs. Another approach involved concurrent IP and systemic chemotherapy and cytoreductive surgery. Yonemura et al. $[21,22]$ and Fujiwara et al. [21, 22] reported that a combined approach with both IP and systemic chemotherapy and cytoreductive surgery increased the efficacy of treatment outcomes in gastric cancer with PC.

Our study had several limitations inherent to retrospective analyses. In terms of treatment characteristics in particular, regimens of chemotherapy and surgery types were not homogeneous across patients or groups. However, we collected information on the natural treatment history of gastric cancer patients with PC without measurable disease, and to our knowledge, this is the first reported evaluation of the clinical characteristics and outcomes of systemic chemotherapy in patients with gastric cancer with PC.

According to the results of our study, we considered that aggressive multimodal treatment, including palliative gastrectomy and/or D1/D2 LN dissection followed by systemic intravenous chemotherapy, was associated with better outcomes in patients with low-grade PC without measurable disease on the preoperative CT scan (these patients were less likely to have metastatic LAP, clinically significant ascites, or bowel involvement), especially when the patients had a good PS. Compared with these patients with low-grade PC, patients with high-grade PC had poor outcomes with the above current practices; thus, it would be better to treat such patients with newer systemic anticancer agents such as anti-angiogenic agents. However, as these different treatment strategies are based not only on clinical characteristics but also on specific molecular characteristics, vigorous study of the molecular characteristics of gastric cancer PC should be performed. In addition, the efficacy of palliative gastrectomy, including cytoreductive surgery and systemic chemotherapy, in patients with low-grade PC without measurable disease should be evaluated separately in prospective trials.

In conclusion, we showed here the clinical characteristics of PC arising from gastric cancer in relation to the clinical grade and outcomes of systemic chemotherapy, especially in patients without measurable disease. And, based on our study, we also suggest that clinical trials in patients with PC arising from gastric cancer should be stratified according to $\mathrm{PC}$ grade.

Acknowledgments This research was supported by Seoul St. Mary's Clinical Medicine Research Program year of 2009 through the Catholic University of Korea.
Conflict of interest None.

\section{References}

1. Bando E, Yonemura Y, Takeshita Y, Taniguchi K, Yasui T, Yoshimitsu Y, et al. Intraoperative lavage for cytological examination in 1,297 patients with gastric carcinoma. Am J Surg. 1999; 178:256-62.

2. Chau I, Norman AR, Cunningham D, Waters JS, Oates J, Ross PJ. Multivariate prognostic factor analysis in locally advanced and metastatic esophago-gastric cancer-pooled analysis from three multicenter, randomized, controlled trials using individual patient data. J Clin Oncol. 2004;22:2395-403.

3. Glehen O, Gilly FN, Boutitie F, Bereder JM, Quenet F, Sideris L, et al. Toward curative treatment of peritoneal carcinomatosis from nonovarian origin by cytoreductive surgery combined with perioperative intraperitoneal chemotherapy: a multi-institutional study of 1,290 patients. Cancer. 2010;116:5608-18.

4. Gill RS, Al-Adra DP, Nagendran J, Campbell S, Shi X, Haase E, et al. Treatment of gastric cancer with peritoneal carcinomatosis by cytoreductive surgery and HIPEC: a systematic review of survival, mortality, and morbidity. J Surg Oncol. 2011;104: 692-8.

5. Yonemura Y, Bandou E, Kawamura T, Endou Y, Sasaki T. Quantitative prognostic indicators of peritoneal dissemination of gastric cancer. Eur J Surg Oncol. 2006;32:602-6.

6. Sadeghi B, Arvieux C, Glehen O, Beaujard AC, Rivoire M, Baulieux J, et al. Peritoneal carcinomatosis from non-gynecologic malignancies: results of the EVOCAPE 1 multicentric prospective study. Cancer. 2000;88:358-63.

7. Ohtsu A, Shah MA, Van Cutsem E, Rha SY, Sawaki A, Park SR, et al. Bevacizumab in combination with chemotherapy as firstline therapy in advanced gastric cancer: a randomized, doubleblind, placebo-controlled phase III study. J Clin Oncol. 2011;29: 3968-76.

8. Franko J, Shi Q, Goldman CD, Pockaj BA, Nelson GD, Goldberg $\mathrm{RM}$, et al. Treatment of colorectal peritoneal carcinomatosis with systemic chemotherapy: a pooled analysis of North Central Cancer Treatment Group Phase III Trials N9741 and N9841. J Clin Oncol. 2012;30:263-7.

9. Therasse P, Arbuck SG, Eisenhauer EA, Wanders J, Kaplan RS, Rubinstein L, et al. New guidelines to evaluate the response to treatment in solid tumors. European Organization for Research and Treatment of Cancer, National Cancer Institute of the United States, National Cancer Institute of Canada. J Natl Cancer Inst. 2000;92:205-16.

10. Lee SS, Lee JL, Ryu MH, Chang HM, Kim TW, Kang HJ, et al. Combination chemotherapy with capecitabine (X) and cisplatin (P) as first line treatment in advanced gastric cancer: experience of 223 patients with prognostic factor analysis. Jpn J Clin Oncol. 2007;37:30-7.

11. Japanese Research Society for Gastric Cancer: Japanese classification of gastric carcinoma. Tokyo: Kanehara; 1995.

12. Sugarbaker PH, Chang D. Results of treatment of 385 patients with peritoneal surface spread of appendiceal malignancy. Ann Surg Oncol. 1999;6:727-31.

13. Blair SL, Chu DZ, Schwarz RE. Outcome of palliative operations for malignant bowel obstruction in patients with peritoneal carcinomatosis from nongynecological cancer. Ann Surg Oncol. 2001;8:632-7.

14. Ross P, Nicolson M, Cunningham D, Valle J, Seymour M, Harper $\mathrm{P}$, et al. Prospective randomized trial comparing mitomycin, cisplatin, and protracted venous-infusion fluorouracil (PVI 5-FU) 
With epirubicin, cisplatin, and PVI 5-FU in advanced esophagogastric cancer. J Clin Oncol. 2002;20:1996-2004.

15. Baba H, Yamamoto M, Endo K, Ikeda Y, Toh Y, Kohnoe S, et al. Clinical efficacy of S-1 combined with cisplatin for advanced gastric cancer. Gastric Cancer. 2003;6(Suppl 1):45-9.

16. Sugarbaker PH, Stuart OA, Carmignani P. Pharmacokinetic changes induced by the volume of chemotherapy solution in patients treated with hyperthermic intraperitoneal mitomycin $\mathrm{C}$. Cancer Chemother Pharmacol 2006;57:703-8.

17. Aoyagi K, Kouhuji K, Yano S, Miyagi M, Imaizumi T, Takeda J, et al. VEGF significance in peritoneal recurrence from gastric cancer. Gastric Cancer. 2005;8:155-63.

18. Ninomiya S, Inomata $M$, Tajima $M$, Ali AT, Ueda $Y$, Shiraishi N, et al. Effect of bevacizumab, a humanized monoclonal antibody to vascular endothelial growth factor, on peritoneal metastasis of MNK-45P human gastric cancer in mice. J Surg Res. 2009;154: 196-202.
19. Masoumi Moghaddam S, Amini A, Morris DL, Pourgholami MH. Significance of vascular endothelial growth factor in growth and peritoneal dissemination of ovarian cancer. Cancer Metastasis Rev. 2012;31:143-62.

20. Yagi Y, Fushida S, Harada S, Tsukada T, Kinoshita J, Oyama K, et al. Biodistribution of humanized anti-VEGF monoclonal antibody/bevacizumab on peritoneal metastatic models with subcutaneous xenograft of gastric cancer in mice. Cancer Chemother Pharmacol. 2010;66:745-53.

21. Yonemura Y, Endou Y, Shinbo M, Sasaki T, Hirano M, Mizumoto A, et al. Safety and efficacy of bidirectional chemotherapy for treatment of patients with peritoneal dissemination from gastric cancer: selection for cytoreductive surgery. J Surg Oncol. 2009;100:311-6.

22. Fujiwara Y, Takiguchi S, Nakajima K, Miyata H, Yamasaki M, Kurokawa Y, et al. Intraperitoneal docetaxel combined with S-1 for advanced gastric cancer with peritoneal dissemination. J Surg Oncol. 2012;105:38-42. 